\title{
Ultrasound Screening in Low-risk Obstetrical Population: Effect on Perinatal Outcome
}

\author{
M Theodora, A Antsaklis \\ Fetal Medicine Unit 1st, Department of Obstetrics and Gynecology \\ University of Athens, Greece \\ Alexandra Hospital
}

\begin{abstract}
The issue of routine sonography for low-risk pregnant women continues to be controversial since randomized trials have not been able to demonstrate a clear benefit especially in prenatal outcome in the past. Ultrasonography early in pregnancy can be definitely used for accurate gestational age determination and detection of multiple gestations. Additionally ultrasound scan is the best method for detecting fetal malformation as well as fetuses in high-risk for growth restriction. Since fetal chromosomal and structural anomalies, prematurity and poor placentation account for $90 \%$ of poor perinatal outcome, ultrasound could theoretically help in decreasing perinatal mortality and severe perinatal morbidity.
\end{abstract}

Key words: Perinatal outcome, screening, ultrasound.

\section{INTRODUCTION}

Antenatal care of pregnant women has been considerably changed since ultrasonography became an important part of routine follow-up in obstetrical practice in many countries, mainly in Europe. Since the 1970s remarkable progress in ultrasound technology along with the growing knowledge and experience of sonographers, enabled obstetrician to reveal some of the well hidden in utero-life secrets.

Fetal chromosomal and structural anomalies, prematurity and poor placentation account for $90 \%$ of poor perinatal outcome. Fetal malformations are the cause of $30 \%$ of perinatal deaths in developed countries, even though their prevalence is relative low, ranging from 2 to $4 \%$ of all births. ${ }^{1}$ Preterm delivery is one of the main causes of perinatal mortality and morbidity and it accounts for 60 to $80 \%$ of deaths of infants without congenital anomalies. ${ }^{2}$

Obstetrical and medical history or conditions such as short cervical length which could be identified in the index pregnancy determine the relative risk for various complications in each pregnancy. In cases of high-risk pregnancies the use of ultrasonography has proved to be of invaluable assistance and with these tests pregnant women do expect to have information about baby's health, the prognosis for the complications as well as the management and the treatment options. In these cases ultrasound examinations are justified and well accepted by the obstetricians since their effect on pregnancy outcome is positive.

The recognition and acceptance of the value of ultrasound tests in high-risk or even complicated pregnancies and the fact that the majority of complications occur in women with no predisposition, have led to evaluation of the utility of ultrasonography in low-risk populations as well. In these cases ultrasound scans are used mainly as screening method. Medical practitioners in other words wondered whether screening pregnant woman with ultrasonography could offer advantages over scanning only those who had firm indications for the test. Screening is the process by which we try to identify in a population who are low-risk for a disease or a medical condition, the subjects that have the disease or that are at increased risk to develop it. In order to introduce a test in clinical practice as a screening method some conditions must be fulfilled. First the condition for which can be offered must be severe enough, with well-known symptoms to be a concern for the community. Second, must be prevalent enough, so that the test to be costefficient. Third, the test should be cheap and easy to perform. Forth, a treatment must be available. Fifth, the sensitivity, specificity and positive predictive value of the screening test must be high enough.

In most European countries, the advantage of ultrasonography in low-risk population has been accepted and two or even three scans are scheduled as part of the routine antenatal care with no regard to risk status. For example, in Germany it is mandated than pregnant women should be offered an ultrasound scan for each trimester and there is an referral system organized in case a fetal anomaly is suspected. ${ }^{3}$ In general, European professional bodies include scans in routine antenatal care of low-risk pregnant women and therefore high compliance rates have been reached. Several consensus conferences took place in Europe and all emphasized the favorable rate of ultrasound examination of low-risk population. Routine use of ultrasound in low pregnancies has been offered in order to decrease labor inductions performed for postdatism, 
as well as for the early detection of multifetal gestations, for detection of placental implantation abnormalities and for the antenatal diagnosis of congenital anomalies.

On the other hand antenatal care seems different in the United States of America and the question of whether or not all pregnant women should have an ultrasound examination continuous to be controversial. The issue of routine use of ultrasonography in low-risk pregnant was addressed in a meeting held by the National Institutes of Health (NIH) on Ultrasound Imaging in Pregnancy in $1984 .{ }^{4}$ The meeting group conducted a list of clinical situations in pregnancy in which ultrasonography might help to improve the outcome but there was no evidence of such a relationship. For example, even if ultrasound scans improved the early detection of multiple gestations and fetal anomalies; there was no improvement in perinatal mortality and severe morbidity. For this reason the working group suggested that "randomized, controlled clinical trials of routine ultrasound screening during pregnancy should be conducted in the United States". Until favorable results for routine use of ultrasonography from such randomized clinical trials are available, examinations should be performed only for specific medical indications during pregnancy.

The RADIUS trial ${ }^{5-7}$ was conducted in order to determine whether routine ultrasound screening decreases the frequency of adverse perinatal outcomes. It was a randomized trial comparing routinely scanned pregnant patients with those having ultrasound only when indicated and up to date it represents one of the largest samples of population studied. In this study which was preformed between 1987 and 1991, 15.151 low-risk pregnant women with no clear indication for an ultrasound examination were randomized in two groups. The patients in the study group were examined at one of the 28 ultrasound laboratories participating in the study. The ultrasound screening group underwent two planned ultrasound examinations: The first at 15 to 22 weeks and the second 31 to 35 weeks. The control group had an ultrasound examination if and when there was an indication for such a test. Fetal death, neonatal death, or neonatal morbidity such as intraventricular hemorrhage were defined as adverse perinatal outcomes. In the control group $60 \%$ of the patients ended up having an ultrasound.

The mean numbers of sonograms obtained per woman in the ultrasound-screening and control groups were 2.2 and 0.6 , respectively. The rate of adverse perinatal outcome was 5.0\% among the infants of the women in the ultrasound-screening group and $4.9 \%$ among the infants of the women in the control group (relative risk, 1.0; 95\% confidence interval, 0.9 to 1.2; $\mathrm{P}=0.85$ ). The rates of preterm delivery and the distribution of birth weights were nearly identical in the two groups. The ultrasonographic detection of congenital anomalies had no effect on perinatal outcome. There were no significant differences between the groups in perinatal outcome in the subgroups of women with postdate pregnancies, multiple-gestation pregnancies, or infants who were small for gestational age.

The writers concluded, that based on their results screening ultrasonography did not improve perinatal outcome as compared with the selective use of ultrasonography on the basis of clinician judgment. ${ }^{5}$ Additionally the financial cost of performing routine ultrasound is a prominent feature in the argument of those who believe that such studies should not be offered to low-risk women. It has been suggested that an additional USD 500 millions per year would be required to provide routine sonography for all pregnant women in the USA. After the publication of this trial the ACOG stated that "in USA the routine use of ultrasound cannot be supported from a cost benefit stand point and that the College does not recommend routine ultrasound screening."

It should, at this point, be noted that a lot of criticism has been made on scientific shortcomings in the methods and therefore the conclusions of the RADIUS trial although it was and remains to be the most expensive and one of the largest prospective studies of the routine versus indicated use of obstetrical ultrasound. One of the main shortcomings of the RADIUS trial is the low detection rate for fetal anomalies which was estimated to be $35 \%$ in contrast with European studies ${ }^{9}$ in which was $56.2 \%$. Furthermore, only $17 \%$ of fetal structural anomalies were detected before 24 weeks, as compared with an average of 52\% (range, 21 to 84\%) in European screening studies. ${ }^{9-14}$ Due to late diagnosis any interventions for malformed fetuses, which could alter the perinatal outcome, were no possible. Another limitation in interpretation of these results is that the study population was at a very low-risk for perinatal problems. Ninety-three percent were white, $70 \%$ had at least some college education, $90 \%$ were in the ideal prepregnancy weight range, only $13 \%$ were smokers, and the mean birth weight of their babies was about $3400 \mathrm{~g}$. This was noted by the writers themselves who recognize, in their conclusion, that routine screening might be more effective in other populations. ${ }^{5}$ Additionally, this trial estimated the effect that routine scans had on perinatal (fetal, neonatal and maternal) death and not on other iatrogenic or biological complications such as postdate pregnancies. In fact there was reported a significant reduction of the number of women with postdate pregnancies in the screened group over the control group245 women and 347 women respectively. This reflected to the reduction of pregnant women at risk for the iatrogenic and biologic complications associated with postdate pregnancies, which is one of the main objectives of routine scanning.

During 1993 three systematic reviews have been published addressing the same subject. The Cochrane Database of Systematic Reviews has published one review of routine prenatal ultrasound examination in early pregnancy ${ }^{15}$ and one 
in late pregnancy. ${ }^{16}$ The review of early prenatal ultrasound trials concluded that screening results in early detection of twin pregnancies, a reduced rate of induction of labor, higher birth weights in singletons and increased rates of abortion for fetal abnormalities, but statistically significant difference on perinatal mortality or Apgar scores was not reported. On the other hand the review of late prenatal ultrasound trials, which did not include the RADIUS trial, concluded that there were no statistically significant effects of late screening on mortality, morbidity or induction of labor.

Another meta-analysis ${ }^{17}$ of four published studies ${ }^{18-21}$ including 15,935 pregnancies also showed that screening with ultrasound did not significantly alter the rate of live birth (Table 1). Nevertheless there was a significantly lower perinatal mortality in the screened population mainly due to the increase of induced abortions of structurally abnormal fetuses. The authors gave also some clinical implications of these results. They noted that "Routine ultrasound scanning in pregnancy is effective in detecting fetal growth retardation, multiple pregnancies, and severe malformations and that it is indicated only if explicitly performed to exclude congenital malformations".

Table 1: Meta-analysis of outcome of pregnancy in randomized controlled trials of routine versus selective ultrasound scanning in pregnancy (Bucher HC, Schmidt JG. BMJ 1993; 307:13-17)

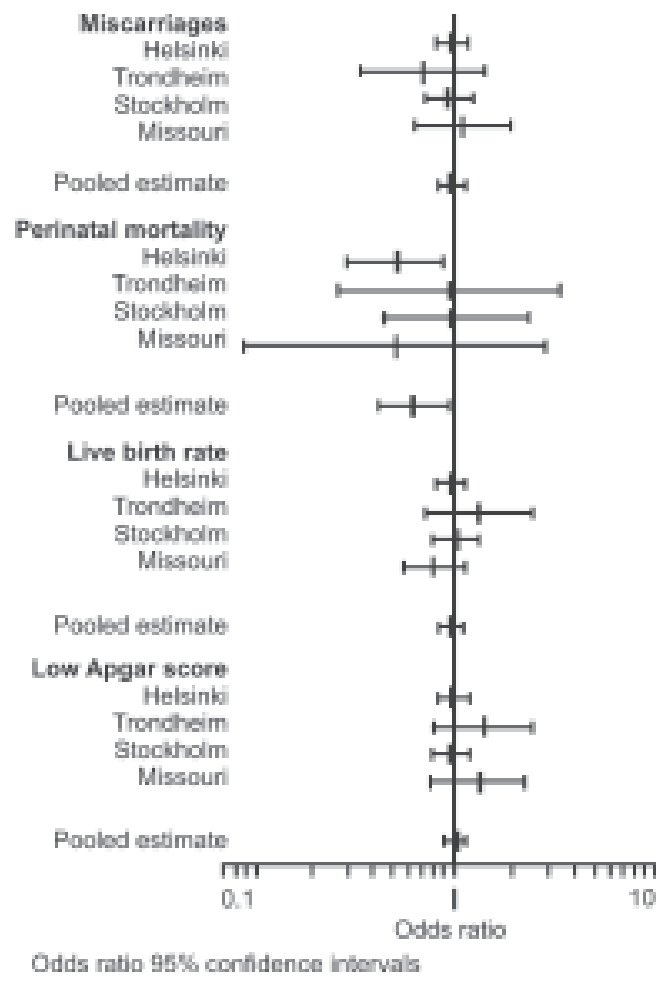

The fact that these meta-analysis and the RADIUS trial have not shown any statistically significant difference in the perinatal outcome in the screened groups is an important information mainly for those who plan health policies and benefits, but these findings should be interpreted with caution. The population, the screening protocol and the detection rate for each anomaly affects the results of a study especially in terms of cost effectiveness. Reported sensitivities and specificities for routine sonography vary widely as a result of factors such as site of examination, length and frequency of examination, number of views obtained, definition of anomalies and outcomes considered.

The past years the use of ultrasound as a screening test for certain obstetrical conditions and complications has been questioned and a considerable number of clinical trials were designed and conducted to answer these questions. The results of these trials confirm that routine ultrasonography can identify the majority of anomalous fetuses and women at high-risk for these conditions which, as mentioned above, account for $90 \%$ of the perinatal mortality.

The principal benefit of routine ultrasonography performed in the middle of the second trimester is the detection of unsuspected fetal anomalies. ${ }^{9}$ Early detection of lethal anomalies provides the parents with the option to terminate the pregnancy. Moreover, detection of structural anomalies in utero should not be thought of only in terms of the termination of pregnancy. This knowledge can be helpful in situations in which prenatal therapy, timely delivery or referral to a tertiary center may improve perinatal outcome and increase the chances of survival. Finally in utero diagnosis may indicate postnatal interventions or closer follow-up of the newborn. ${ }^{22,23}$

Preterm delivery is another complication which cause adverse perinatal outcome. The use of cervical assessment with ultrasound has been well established. It is a widely accepted and well-standardized method which can be easily performed in both high and low-risk patients as a screening test for preterm delivery. ${ }^{24}$ It has a high negative predictive value in a high-risk population but because the low prevalence of preterm delivery, the sensitivity and positive predictive value are low in a lowrisk population. Nevertheless it is a better method than obstetric history in predicting preterm labor and when used together the detection rate is almost $60 \% .{ }^{25}$ The risk of premature birth in women with short celvical length (less than $15 \mathrm{~mm}$ ) can be reduced with prophylactic administration of progesterone from midgestation to 34 weeks. ${ }^{26-28}$ Reduction of preterm delivery could have a positive impact on perinatal mortality and severe morbidity since $50 \%$ of deaths in fetuses with no congenital anomalies are due to prematurity.

Routine ultrasonography has been also used in early diagnosis and screening obstetric complications which emerge from abnormal placentation such as pre-eclampsia and intrauterine growth restriction. Increased impedance to flow in 
the uterine arteries in both high-risk and low-risk pregnancies is associated with increased risk for subsequent development of pre-eclampsia and intrauterine growth restriction. Increased impedance to flow in the uterine arteries at 23 weeks of gestation is found in about $5 \%$ of pregnancies attending for routine antenatal care and identifies about $30 \%$ of those that subsequently develop intrauterine growth restriction. Abnormal Doppler is better in predicting severe conditions rather than the mild. Implementation of a uterine artery screening program into routine antenatal care perhaps at the time of the 2nd trimester anomaly scan would help stratify the intensity of subsequent surveillance. The questions to be answered by future research is whether there could be any useful intervention in order to reduce poor perinatal outcome since neither pharmacological intervention in the second trimester nor close surveillance have been proved to alter the perinatal outcome of severe cases.

\section{CONCLUSION}

Ultrasonography in pregnancy improves the dating of gestational age, ${ }^{29}$ which may lead to a reduction in the number of induced labors in cases in which the gestation is over estimated. ${ }^{18,30} \mathrm{It}$ is the most accurate means of detecting retardation in fetal growth $^{31}$ and multiple gestations ${ }^{32}$ and it is effective in detecting severe malformations.

Large population studies and different critical reviews have failed to prove that routine ultrasound in pregnancy effectively alter perinatal outcome in terms of fetal and neonatal death and severe morbidity. But this maybe due to many reasons such as lack of clear and consistent definition of sonographic examination needed and clearly defined outcomes. Nevertheless these trials have showed that ultrasound screening in low-risk population reduce perinatal mortality as the result of induced abortions following the detection of abnormalities. Most recent trials have established that ultrasound screening when preformed based on a realistic schedule and by trained sonographers can identify the majority of malformed fetuses and pregnant women who may be at risk for the two conditions which are responsible for the majority of perinatal deaths: Pre-eclampsia and preterm delivery.

Despite these achievements, however, the important question is whether routine ultrasonography improves the outcome of pregnancy and whether it shows an overall net benefit still stands. Research to determine the advantage of screening ultrasound should continue and future investigations should be designed with carefully defined screening protocols and outcome criteria in order to be interpreted in to clinical application for the benefit of the pregnant women and their babies.

\section{REFERENCES}

1. Hobbins JC, Grannum PAT, Berkowitz RL, Silverman R, Mahoney MJ. Ultrasound in the diagnosis of congenital anomalies, 1979.

2. McCormack MC. The contribution of low birth weight to infant mortality and childhood morbidity. N Engl J Med 1985;312:8290.

3. Salvator Levi. Ultrasound in prenatal diagnosis: Polemics around routine ultrasound screening for second trimester fetal malformations. Prenat Diagn 2002;22:285-95.

4. Office of Medical Applications of Research, National Institutes of Health. The use of diagnostic ultrasound imaging during pregnancy. JAMA 1984;252:669-72.

5. Ewigman BG, Crane JP, Frigoletto FD, LeFevre ML, Bain RP, McNellis D. The RADIUS Study Group Effect of Prenatal Ultrasound Screening on Perinatal Outcome. NEJM 329(12):821-27

6. Crane JP, LeFevre ML, Winborn RC, et al. RADIUS Study group. A randomized trial of prenatal ultrasonographic screening: Impact on the detection, management, and outcome of anomalous fetus. Am J Obstet Gynecol 1994;171:392-99.

7. LeFevre ML, Bain RP, Ewigman BG, Frigoletto FD, Crane JP, Mcnellis D. A randomized trial of prenatal ultrasonographic screening: Impact on maternal management and outcome. Am J Obstet Gynecol 1993;169:483-89.

8. ACOG practice patterns. Routine ultrasound in low-risk pregnancies. Int J Gynecol Obstet 1997;59:273-78.

9. Grandjean H, Larroque D, Levi S, and the Eurofetus team. The performance of routine ultrasonographic screening of pregnancies in the Eurofetus study. Am J Obstet Gynecol 1999;181:446-54.

10. Rosendahl H, Kivinen S. Antenatal detection of congenital malformations by routine ultrasonography. Obstet Gynecol 1989;73:947-51.

11. Levi S, Hyjaz Y, Schaaps JP, Defoort P, Coulon R, Buekens P. Sensitivity and specificity of routine antenatal screening for congenital anomalies by ultrasound: The Belgian Multicentric Study. Ultrasound Obstet Gynecol 1991;1:102-10.

12. Chitty LS, Hunt GH, Moore J, Lobb MO. Effectiveness of routine ultrasonography in detecting fetal structural abnormalities in a low-risk population. BMJ 1991;303:116569.

13. Luck CA. Value of routine ultrasound scanning at 19 weeks: A four year study of 8849 deliveries. BMJ 1992;304:1474-78.

14. Shirley IM, Bottomley F, Robinson VP. Routine radiographer screening for fetal abnormalities by ultrasound in an unselected low-risk population. Br J Radiol 1992;65:564-69.

15. Neilson JP. Routine ultrasound in early pregnancy. Pregnancy and Childbirth Module. In Enkin MW, Keirse MJNC, Renfrew MJ, Neilson JP (Eds): "Cochrane Database of Systematic Reviews”: Review No. 03872, 9 June 1993. Published through “Cochrane Updates on Disk”, Oxford: Update Software, 1994, Disk Issue 1. 
16. Neilson JP. Routine fetal anthropometry in late pregnancy. Pregnancy and Childbirth Module. In Enkin MW, Keirse MJNC, Renfrew MJ, Neilson JP (Eds): “Cochrane Database of Systematic Reviews”: Review No. 03873, 24 March 1993. Published through “Cochrane Updates on Disk”, Oxford: Update Software, 1994, Disk Issue 1.

17. Bucher HC, Schmidt JG. Does routine ultrasound screening improve outcome in pregnancy? Meta-analysis of various outcome measures. BMJ 1993;307:13-17.

18. Waldenstrom U, Axelsson O, Nilsson S, Eklund G, Fall O, Lindenberg S, et al. Effects of routine one-stage ultrasound screening in pregnancy: A randomized controlled trial. Lancet 1988;ii:385-88.

19. Saari-Kemppainen A, Karialainen O, Ylostalo P, Heinonen OP. Ultrasound screening and perinatal mortality: Controlled trial of systematic one-stage screening in pregnancy. The Helsinki Ultrasound Trial. Lancet 1990;336:387-91.

20. Bakketeig LS, EikNes SH, Jacobsen G, Ulstein GK, Brodtkorb CJ, Balstad P, et al. Randomized controlled trial of ultrasonographic screening in pregnancy. Lancet 1984;207-11.

21. Ewigman B, LeFevre M, Hesser J. A randomized trial of routine prenatal ultrasound. Obstet Gynecol 1990;76:189-94.

22. Hajdu J, Marton T, Toth PE. Congenital heart abnormalities and cardiac dysfunction: How prenatal diagnosis hanged the chances for survival. Orv Hetil 140:815-18.

23. Gotoh H, Masuzaki H, Fukuda H, Yoshimura S, Ishimaru T. Detection and assessment of pyelectasis in the fetus: Relationship to postnatal renal function. Obstet Gynecol 92:226-31.

24. To MS, Skentou CA, Royston P, Yu CK, Nicolaides KH. Prediction of patient-specific risk of early preterm delivery using maternal history and sonographic measurement of cervical length: A population-based prospective study. Ultrasound Obstet Gynecol 2006;27:362-67.

25. Nicolaides KH. Some thoughts on the true value of Ultrasound (Editorial) Ultrasound Obstet Gynecol 2007;30: 671-74.

26. Fonseca EB, Bittar RE, Carvalho MH, Zugaib M. Prophylactic administration of progesterone by vaginal suppository to reduce the incidence of spontaneous preterm birth in women at increased risk: A randomized placebo-controlled double-blind study. Am J Obstet Gynecol 2003;188:419-24.

27. Fonseca EB, Celik E, Parra M, Singh M, Nicolaides KH. Progesterone and the risk of preterm birth among women with a short cervix. N Engl J Med 2007;357:462-69.

28. Clement S, Candy B, Heath V, To M, Nicolaides KH. Transvaginal ultrasound in pregnancy: Its acceptability to women and maternal psychological morbidity. Ultrasound Obstet Gynecol 2003;22:508-14.

29. Campell S, Warsof SL, Little D, Cooper DJ. Routine ultrasound screening for the prediction of gestational age. Obstet Gynecol 1985;65:613-20.

30. Persson PH, Kullander S. Long-term experience of general ultrasound screening in pregnancy. Am J Obstet Gynecol 1983;146:942-47.

31. Mintz MC, Landon MB. Sonographic diagnosis of fetal growth disorders. Clin Obstet Gynecol 1988;31:44-52.

32. Grennert L, Persson PH, Gennser G. Benefits of ultrasonic screening of a pregnant population. Acta Obstet Gynecol Scan Suppl 1978;78:5-14. 